\title{
Resting and reactive frontal brain electrical activity (EEG) among a non-clinical sample of socially anxious adults: Does concurrent depressive mood matter?
}

\author{
Elliott A Beaton' \\ Louis A Schmidt ${ }^{2}$ \\ Andrea R Ashbaugh ${ }^{2,5}$ \\ Diane L Santesso ${ }^{2}$ \\ Martin M Antony ${ }^{1,3,4}$ \\ Randi E McCabe ${ }^{1,3}$ \\ ${ }^{1}$ Department of Psychiatry and \\ Behavioural Neurosciences, McMaster \\ University, Hamilton, Ontario, \\ Canada; ${ }^{2}$ Department of Psychology, \\ Neuroscience and Behaviour, \\ McMaster University, Hamilton, \\ Ontario, Canada; ${ }^{3}$ Anxiety Treatment \\ and Research Centre, St. Joseph's \\ Healthcare, Hamilton, Ontario, \\ Canada; ${ }^{4}$ Department of Psychology, \\ Ryerson University, Toronto, Ontario, \\ Canada; ${ }^{5}$ Concordia University, \\ Montreal, Quebec, Canada
}

Correspondence: Louis A Schmidt Department of Psychology, Neuroscience and Behaviour, McMaster University, Hamilton, Ontario L8S 4KI, Canada Tel + I 9055259140 ext 23028 $\mathrm{Fax}+$ I 9055296225

Email schmidtl@mcmaster.ca

\begin{abstract}
A number of studies have noted that the pattern of resting frontal brain electrical activity (EEG) is related to individual differences in affective style in healthy infants, children, and adults and some clinical populations when symptoms are reduced or in remission. We measured self-reported trait shyness and sociability, concurrent depressive mood, and frontal brain electrical activity (EEG) at rest and in anticipation of a speech task in a non-clinical sample of healthy young adults selected for high and low social anxiety. Although the patterns of resting and reactive frontal EEG asymmetry did not distinguish among individual differences in social anxiety, the pattern of resting frontal EEG asymmetry was related to trait shyness after controlling for concurrent depressive mood. Individuals who reported a higher degree of shyness were likely to exhibit greater relative right frontal EEG activity at rest. However, trait shyness was not related to frontal EEG asymmetry measured during the speech-preparation task, even after controlling for concurrent depressive mood. These findings replicate and extend prior work on resting frontal EEG asymmetry and individual differences in affective style in adults. Findings also highlight the importance of considering concurrent emotional states of participants when examining psychophysiological correlates of personality.
\end{abstract}

Keywords: social anxiety, shyness, sociability, depression, mood, frontal EEG alpha asymmetry

Recent studies have found that the pattern of resting frontal EEG alpha asymmetry is predictive of individual differences in affective style in healthy adults and children, some clinically depressed and anxious populations even when their symptoms are in remission (for reviews see Davidson 1993, 2000; Davidson et al 2003; Coan and Allen 2004), and adults with schizophrenia whose negative symptoms are reduced (Jetha et al in press). Normal adults (Sutton and Davidson 1997; Schmidt 1999) and children (for reviews see Fox 1991; Fox et al 2001) who exhibit right frontal EEG asymmetry at rest are easily distressed, fearful, and shy, whereas those who exhibit left frontal EEG asymmetry at rest are socially outgoing and extraverted. Because the pattern of frontal EEG asymmetry at rest is stable across time (Tomarken et al 1992) and context (Schmidt et al 2003) and its appearance early in life is predictive of later personality (Fox et al 2001), some have argued that this metric represents a "trait-like" marker of dispositional affective style (Davidson 1993, 2000). Here we extend these earlier findings of studies with normal individuals by examining whether the pattern of frontal EEG alpha asymmetry at rest and in response to an anticipated speech distinguished among individual differences in social anxiety in a non-clinical sample of young adults. 
We previously found that a non-clinical sample of adults who were selected for high and low social anxiety (ie, high and low shyness) displayed greater relative right frontal EEG activity at rest (Schmidt 1999). Others have found greater relative right frontal EEG activity in a clinical group of socially phobic adults during anticipation of public speaking (Davidson et al 2000). We also noted an increase in right, but not left, frontal EEG activity in typically developing shy children in anticipation of a social speech (Schmidt et al 1999), but failed to find this relation in a non-clinical sample of shy and socially anxious adults in response to anticipated social interaction (Schmidt and Fox 1994).

One possible reason for these inconsistencies among earlier studies may be the failure to consider concurrent mood. For example, disparate studies have noted relations between depression and right frontal brain activity at rest (Henriques and Davidson 1990,1991) as well as between depression and shyness (Schmidt and Fox 1995), but there appear to be no studies that have examined the possibility that concurrent depressive mood may influence the predicted shy-right frontal EEG asymmetry relation in non-clinical participants.

In the present study, participants included a healthy, nonclinical sample of young adults selected for high (HSA) and low (LSA) social anxiety. Regional brain electrical activity (EEG) was indexed both at rest and while participants were preparing a speech in anticipation of being videotaped for others viewing. Participants also completed self-report measures of trait shyness and sociability and concurrent depressive mood. We predicted that the HSA group would exhibit greater relative right frontal EEG asymmetry at rest and during the speech preparation task than the LSA group. We also predicted that shyness would be related to greater relative right frontal EEG activity at rest and during the speech preparation task, while sociability would be related to greater relative left frontal EEG activity at rest and in response to the speech task, regardless of group, after controlling for concurrent depressive mood.

\section{Method}

\section{Participants}

Participants were 330 ( 84 males, 246 females; $M$ age $=20.32$ years, $S D=4.18$ years) undergraduate students enrolled in psychology courses at McMaster University who completed a series of prescreening questionnaires and received academic credit for their participation.

\section{Participant selection}

Of the 330 participants, 90 scored \pm 1 SD above or below the mean on the Social Phobia Inventory (SPIN). These 90 indi- viduals were contacted to participate in the study. Of these 90 participants, 10 were excluded because they were left-handed (Heller and Levy 1981). Of the remaining 80, 49 (12 males, 37 females) agreed to participate in the study: 24 high socially anxious (HSA; 5 males, 19 females) and 25 low socially anxious (LSA; 7 males, 18 females). There were no significant differences in age, sex, or ethnicity distribution between the two groups. All participants were healthy with no history of psychiatric or neurological problems. Participants were given more academic credit points, a chance to win a $\$ 75$ gift certificate from the university bookstore, or $\$ 10$ cash for their participation.

\section{Self-report measures}

\section{Social Phobia Inventory (SPIN)}

The Social Phobia Inventory (SPIN) (Connor et al 2000) is a 17 -item self-report measure of fear, avoidance, and physiological symptoms associated with social phobia and more generally social anxiety. The SPIN specifically focuses upon fears of talking and socializing with others, and fears of experiencing and exhibiting physiological signs of anxiety such as blushing or sweating (eg, "I avoid giving speeches"). The SPIN has demonstrated good test-retest reliability, good internal consistency, convergent validity, divergent validity, and construct validity in clinical and non-clinical samples (Connor et al 2000; Antony et al 2006; Radomsky et al 2006).

\section{Cheek and Buss Shyness and Sociability Scale}

The five highest loaded items (see Bruch et al 1989) from the 13-item revised Cheek and Buss Shyness Scale (Cheek 1983) were used to assess shyness (eg, "I find it hard to talk to strangers"). Sociability was assessed using the 5 -item Cheek and Buss Sociability Scale (1981) (eg, "I find people more stimulating than anything else"). Reliability and validity data for the two scales are provided elsewhere (Cheek and Buss 1981; Bruch et al 1989).

\section{Depression Anxiety Stress Scales: 2 I-item version (DASS-2I)}

The DASS-21 (Lovibond and Lovibond 1995) is a 21item self-report measure designed to distinguish among depression, anxiety, and symptoms of general tension. The depression scale includes items that evaluate feelings of sadness and worthlessness (eg, "I couldn't seem to experience any positive feeling at all"). The anxiety scale includes items that evaluate symptoms of physical arousal and fear (eg, "I was worried about situations in which I might panic and make a fool of myself"). The stress scale includes items 
that evaluate symptoms of general tension and irritability (eg, "I felt that I was using a lot of nervous energy"). The DASS21 has good reliability and good concurrent validity in both clinical and community samples (Antony et al 1998).

\section{Procedure}

All participants were tested at the Child Emotion Laboratory at McMaster University, Hamilton, Ontario. All procedures were approved by the McMaster University Research Ethics Board. Participants were asked to refrain from consuming excessive amounts of alcohol 24 hours prior to the study and to refrain from smoking or consuming any caffeine two hours prior to the study. Upon the participants' arrival to the laboratory, informed consent was obtained, and participants were asked if they consumed any nicotine or caffeine within the previous two hours. No participant reported having either nicotine or caffeine two hours prior to arrival or alcohol in the previous 24 hours. All participants were medication free and reported that they were not experiencing any extraordinarily stressful life events (eg, recent death in family or prolonged illness). All participants were tested between 1600 and 2000 hours. Regional EEG was continuously recorded for two minutes ( 1 minute eyes-open and 1 minute eyes-closed) after completion of the consent form and after the participants had a chance to acclimatize to the laboratory setting (ie, Time 1: Baseline).

Participants were then told that they were to prepare for a self-presentation task. Specifically, they were told the following:

\footnotetext{
"We are interested in your opinions about classroom presentations. For example, do you think that classroom presentations reflect the true ability of students? Are they useful learning experiences for students? We would like you to give a brief 3 minute discussion that will be videotaped and shown to some participants at a later date. We will give you 2 minutes to prepare your discussion; please make your discussion as close to 3 minutes as possible."
}

Participants were then given 2 minutes to prepare their presentation, during which time regional EEG was continuously recorded (ie, Time 2: Speech Preparation Task). Following the EEG recording, the cap and electrodes were removed, and the participants were asked to give the 3 minute speech. Following the speech, participants were debriefed and thanked.

\section{EEG data collection and data reduction EEG recording}

EEG was recorded using a lycra stretch cap (Electro-Cap, Inc.) which has electrodes sewn into it and positioned according to the International 10/20 Electrode System (Jasper 1958). An abrasive electrolyte gel (Omni-prep) was applied to each electrode site with a cotton swab to increase conductivity. Electrode impedances were all below $10 \mathrm{k} \Omega$ and within $500 \Omega$ between homologous sites. Regional EEG was recorded from four locations: left and right mid-frontal (F3, F4) and parietal $(\mathrm{P} 3, \mathrm{P} 4)$ regions, representing the left and right hemispheres and the anterior and posterior regions of the brain. EEG data were recorded from the parietal region to ensure that the effects were specific to the frontal sites. Electrodes were referenced to the central vertex $(\mathrm{Cz})$ during recording. EEG signals were amplified using SA Instrumentation Bioamplifiers, with bandpass filters set at $1 \mathrm{~Hz}$ (high pass) and $100 \mathrm{~Hz}$ (low pass). Data from each channel were digitized on-line at a sampling rate of $512 \mathrm{~Hz}$.

\section{EEG data reduction and analysis}

EEG data were visually scored for artifact $( \pm 50 \mu \mathrm{V})$ resulting from eye blinks, eye movements, or other motor movements using the EEG Analysis Program (James Long Company, Caroga Lake, NY, USA), which removes artifact data from all channels if artifacts are detected on any one channel. Artifactedited data were further examined to ensure that they did not vary systematically between participants. Artifact-free epochs were analyzed using a discrete Fourier transform (DFT), with a Hanning window of one second width and $50 \%$ overlap. Power (microvolts squared) was derived from the DFT output in the alpha ( 8 to $13 \mathrm{~Hz}$ ) frequency band. A natural $\log (\ln )$ of EEG power was then performed for power at each of the four sites in order to reduce skewness. Separate mid-frontal and parietal EEG asymmetry scores were calculated as follows: frontal asymmetry $=[\ln (\mathrm{F} 4)$ minus $\ln (\mathrm{F} 3)]$; and parietal asymmetry $=[\ln (\mathrm{P} 4)$ minus $\ln (\mathrm{P} 3)]$. Because EEG power is inversely related to activity, resulting negative values reflect greater relative right hemisphere activity (Davidson and Tomarken 1989). Usable baseline (resting) EEG data were available on 41 participants, and usable task (reactive) EEG data were available on 39 participants, owing to equipment problems, excessive artifacts, or refusal to wear the cap. The HSA and LSA groups did not differ in EEG data loss.

\section{Results}

A repeated measures analysis of variance (ANOVA) with Group (HSA, LSA) as a between-subjects factor and Condition (Baseline, Speech Preparation Task) as a withinsubjects factor was performed on the separate frontal and parietal EEG alpha asymmetry scores. Contrary to our prediction, the HSA and LSA groups did not differ on 
(A) Baseline

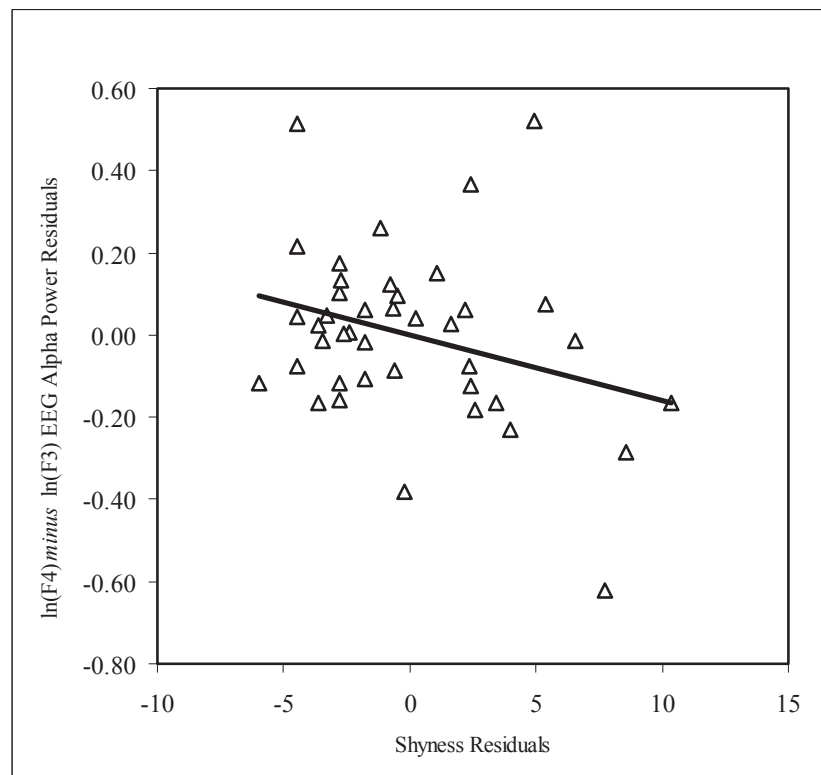

(B) Speech Preparation Task

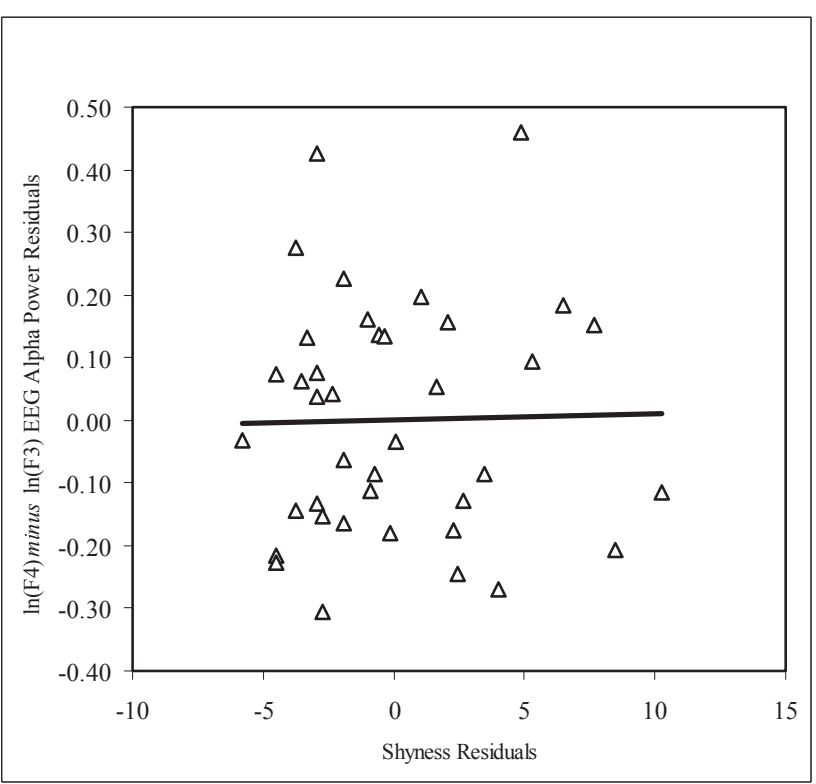

Figure I Residuals scatterplots of the relation between trait shyness (as measured by the Cheek and Buss Shyness Scale) and (A) baseline mid-frontal EEG alpha power asymmetry and (B) during the speech preparation task in a non-clinical sample of healthy young adults after controlling for concurrent depressive mood (Note: Negative EEG asymmetry scores reflect greater relative right frontal EEG activity.)

resting (ie, Time 1: Baseline) or reactive (ie, Time 2: Speech Preparation Task) frontal EEG asymmetry. We next examined the relation between resting and reactive frontal and parietal EEG asymmetry scores separately in relation to shyness and sociability, given previous results that these traits are related to the pattern of resting (Schmidt 1999) and reactive (Schmidt et al 1999) frontal EEG asymmetry. A series of zero-order Pearson correlations were performed among the resting and reactive frontal and parietal EEG asymmetry scores and shyness and sociability measures. Contrary to our prediction, these relations were also not significant.

We then performed post-hoc analyses to examine whether concurrent depressive mood may have influenced the relations between resting and reactivity frontal EEG asymmetry and shyness and sociability, given that depressed individuals are known to exhibit distinct patterns of resting frontal brain activity (Henriques and Davidson 1990, 1991). Partial correlation coefficients were calculated between the shyness and sociability measures and frontal and parietal EEG asymmetry scores at baseline and during the speech preparation task after controlling for scores on the DASS depressive mood scale. As predicted, higher levels of self-reported shyness predicted greater relative right frontal EEG asymmetry at baseline only after controlling for concurrent depressive mood $(r=-0.30, p=0.03)$ (see Figure 1A). However, sociability did not correlate with frontal EEG asymmetry at baseline even after controlling for concurrent depressive mood $(r=0.08, n s)$. Contrary to our prediction, neither shyness nor sociability was related to frontal EEG asymmetry during the speech preparation task even after controlling for depressive $\operatorname{mood}(p s>0.05)$. In addition, there were no significant relations between the resting and reactive parietal EEG asymmetry measures and shyness and sociability scores.

\section{Discussion}

Are there distinct patterns of resting and reactive frontal brain activity among socially anxious adults? Apparently, the answer to this question depends on the level of concurrent depressive mood. We found the predicted relation between shyness and right frontal EEG asymmetry at rest only after controlling for concurrent depressive mood. Individuals who self-reported a high degree of shyness exhibited greater relative right frontal EEG activity at rest after controlling for concurrent depressive mood. This finding is consistent with other recent studies that have shown concurrent symptoms may obscure the predicted relation between personality/ affective style and resting frontal brain activity (Jetha et al in press).

There are, however, a number of limitations that need to be discussed. First, the results of the present study were based on a relatively small sample size of non-clinical undergraduates. These data need to be replicated with larger nonclinical university and community samples and a clinical 
sample in order to ensure the reliability and generalizability of these results. Second, there is typically a high degree of co-morbidty between anxiety and depression. Given this comorbidity, the results may have been influenced not solely by depressed mood. Although the anxiety and stress subscales of the DASS were examined, only the depression subscale influenced the relation between trait shyness and the pattern of frontal EEG asymmetry at rest. Nevertheless, future work should examine different subtypes of co-morbid anxiety and depression with a larger sample size in order to partial out separate and joint influences of anxiety and depressive mood in relation to personality traits and the pattern of frontal EEG activity at rest. Third, we failed to find relations between the selected groups of high and low socially anxious adults. It may be that the limited number of participants in the present study contributed to the lack of statistical power needed to detect between group differences. Alternatively, the extreme group design may not have provided the variability needed to detect differences while the continuous measure of shyness did. Fourth, we failed to find differences on the task data. Again, the lack of findings may have been due to the fact that the study was statistically underpowered to detect differences, the task itself was not robust enough to elicit anxiety, or reactive frontal EEG asymmetry measures play different roles in trait shyness and anxious states. EEG asymmetries may play different roles in pathological and non-pathological disorders and states.

The results of the present study have theoretical and clinical implications. Our results suggest that concurrent depressive mood influences the relation between resting frontal brain activity and individual differences in affective style. It is possible that the predicted relations between personality traits and resting frontal EEG asymmetry may be obscured due to concurrent mood states. Accordingly, it is important for future studies to consider concurrent mood states when examining relations between personality traits and frontal EEG asymmetry measures. The results also have clinical relevance to studies that may use the frontal EEG asymmetry measures as a potential efficacy measure of pre- and post-treatment effects. That is, changes in frontal EEG asymmetry may be coincident with certain therapies used to treat mood and anxiety disorders, and the extent of change may reflect the effectiveness of the therapy. Future studies should extend the current findings to a larger sample of adults clinically diagnosed with social phobia and examine the influence of other co-morbid concurrent anxiety problems on the pattern of resting and reactive frontal brain activity.

\section{Acknowledgments}

This research was supported in part by grants from the Social Sciences and Humanities Research Council of Canada (SSHRC) awarded to Louis A. Schmidt and Martin M. Antony. Portions of this paper were based on the third author's undergraduate honours thesis submitted in partial fulfillment of the Bachelor of Art degree conducted under the direction of LAS, MMA, and REM and presented at the 32nd Annual Ontario Undergraduate Psychology Thesis Conference. The authors would like to acknowledge Sylvia Nowakowski, Austina Reed, and Kate Spere for their help with data collection.

\section{References}

Antony MM, Bieling P, Cox BJ, et al. 1998. Psychometric properties of the 42-time and 21-item versions of depression anxiety stress scales in clinical groups and a community sample. Psychological Assessment, 10:176-81.

Antony MM, Coons MJ, McCabe RE, et al. 2006. Psychometric properties of the Social Phobia Inventory: Further evaluation. Behaviour Research and Therapy, 44:1177-85.

Bruch MA, Gorsky JM, Collins TM, et al. 1989. Shyness and sociability reexamined: A multicomponent analysis. Journal of Personality and Social Psychology, 57:904-15.

Cheek JM. 1983. The revised Cheek and Buss Shyness Scale. Unpublished manuscript. Wellesley College.

Cheek JM, Buss AH. 1981. Shyness and sociability. Journal of Personality and Social Psychology, 41:330-9.

Coan JA, Allen JJB. 2004. Frontal EEG asymmetry as a moderator and mediator of emotion. Biological Psychology, 67:7-49.

Connor KM, Davidson JRT, Churchill LE, et al. 2000. Psychometric properties of the social phobia inventory (SPIN). British Journal of Psychiatry, 176:379-86.

Davidson RJ. 1993. The neuropsychology of emotion and affective style. In Lewis M, Haviland JM eds. Handbook of emotion. New York: Guilford.p 143-54.

Davidson RJ. 2000. Affective style, psychopathology, and resilience: Brain mechanisms and plasticity. American Psychologist, 55:1196-214.

Davidson RJ, Marshall JR, Tomarken AJ, et al. 2000. While a phobic waits: Regional brain electrical and autonomic activity in social phobics during anticipation of public speaking. Biological Psychiatry, 47:85-95.

Davidson RJ, Scherer KR, Goldsmith HH eds. 2003. Handbook of affective science. New York: Oxford University Press.

Davidson RJ, Tomarken AJ. 1989. Laterality and emotion: An electrophysiological approach. In Boller F, Grafman J eds. Handbook of neuropsychology. New York: Elsevier. p 419-41.

Fox NA. 1991. If it's not left, it's right: Electroencephalogram asymmetry and the development of emotion. American Psychologist, 46:863-72.

Fox NA, Henderson HA, Rubin KH, et al. 2001. Continuity and discontinuity of behavioral inhibition and exuberance: Psychophysiological and behavioral influences across the first four years of life. Child Development, 72:1-21.

Heller W, Levy J. 1981. Perception and expression of emotion in righthanders and left-handers. Neuropsychologia, 19:263-72.

Henriques JB, Davidson RJ. 1990. Regional brain electrical asymmetries discriminate between previously depressed and healthy control subjects. Journal of Abnormal Psychology, 99:22-31.

Henriques JB, Davidson RJ. 1991. Left frontal hypoactivation in depression. Journal of Abnormal Psychology, 100:535-45.

Jasper HH. 1958. The ten-twenty electrode system of the International Federation. Electroencephalography and Clinical Neurophysiology, 10:371-75. 
Jetha MK, Schmidt LA, Goldberg JO. In press. Resting frontal EEG asymmetry and shyness and sociability in schizophrenia: A pilot study of community-based outpatients. International Journal of Neuroscience

Lovibond PF, Lovibond SH. 1995. The structure of negative emotional states: Comparisons of the Depression Anxiety Stress Scales (DASS) and the Beck Depression Inventories. Behavior Research and Therapy, 33:335-43.

Radomsky AS, Ashbaugh AR, Saxe ML, et al. 2006. Psychometric properties of the French and English versions of the Social Phobia Inventory. Canadian Journal of Behavioural Science, 38:354-60.

Schmidt LA. 1999. Frontal brain electrical activity in shyness and sociability. Psychological Science, 10:316-20.

Schmidt LA, Cote KA, Santesso DL, et al. 2003. Frontal electroencephalogram alpha asymmetry during sleep: Stability and its relation to affective style. Emotion, 3:401-7.
Schmidt LA, Fox NA. 1994. Patterns of cortical electrophysiology and autonomic activity in adults' shyness and sociability. Biological Psychology, 38:183-98.

Schmidt LA, Fox NA. 1995. Individual differences in young adults' shyness and sociability: Personality and health correlates. Personality and Individual Differences, 19:455-62.

Schmidt LA, Fox NA, Schulkin J, et al. 1999. Behavioral and psychophysiological correlates of self-presentation in temperamentally shy children. Developmental Psychobiology, 35:119-35.

Sutton SK, Davidson RJ. 1997. Prefrontal brain asymmetry: A biological substrate of the behavioral approach and inhibition systems. Psychological Science, 8:204-10.

Tomarken AJ, Davidson RJ, Wheeler RE, et al. 1992. Psychometric properties of resting anterior EEG asymmetry: Temporal stability and internal consistency. Psychophysiology, 29:576-92. 